\title{
Mobile robot guidance using light dependant resistor device
}

\begin{abstract}
The purpose of this study is to develop a mobile robot which is able to be guided by a light source illuminated on the horizontal surface. The robot is equipped with light dependant resistors to detect the spot of light illuminated by the light source which has higher light intensity than the ambient lighting. In particular, we investigated the voltage response of the light dependant resistor given various positioning of the device on the mobile robot and verified the experimental data with the lux meter reading. Results show that the detection distance is a strong function of the facing angle of the light dependant resistor and detection distance for the robot is $50 \mathrm{~cm}$ for facing angle of 0 degrees.
\end{abstract}

Keyword: Light dependant resistor; Light intensity; Lux meter; Mobile robot guidance 